\title{
Accelerating Implementation of Virtual Care in an Integrated Health Care System: Future Research and Operations Priorities
}

\author{
Allison A. Lewinski, PhD, MPH ${ }^{1,2,3} \mathbb{B}^{\mathrm{D}}$, Caitlin Sullivan, $B S^{7}$, Kelli D. Allen, $P \mathrm{PD}^{1,4}$, \\ Matthew J. Crowley, MD, MHS ${ }^{1,5}$, Jennifer M. Gierisch, $P h D, M P H^{1,6,7}$, \\ Karen M. Goldstein, MD, MSPH ${ }^{1,7}$, Kaileigh Gray, BS ${ }^{7}$, Susan N. Hastings, MD, MHS ${ }^{1,6,8,9}$, \\ George L. Jackson, PhD, MHA ${ }^{7,6,7}$, Felicia McCant, MSSW' , Abigail Shapiro, MSPH', \\ Matthew Tucker, BS ${ }^{7}$, Carolyn Turvey, PhD 10,11,12, Leah L. Zullig, PhD 1,6, and \\ Hayden B. Bosworth, $P h D^{1,2,6,7,9,13,14}$
}

\begin{abstract}
'Durham Center of Innovation to Accelerate Discovery and Practice Transformation, Durham Veterans Affairs Health Care System, Durham, NC, USA; ${ }^{2}$ School of Nursing, Duke University, Durham, NC, USA; ${ }^{3}$ HSR\&D COIN (558/152), Durham Veterans Affairs Health Care System, 508 Fulton Street, Durham, NC, USA; ${ }^{\circ}$ Department of Medicine \& Thurston Arthritis Research Center, University of North Carolina at Chapel Hill, Chapel Hill, NC, USA; ${ }^{5}$ Division of Endocrinology, Diabetes and Metabolism, Department of Medicine, Duke University School of Medicine, Durham, NC, USA; ${ }^{6}$ Department of Population Health Sciences, Duke University School of Medicine, Durham, NC, USA; ${ }^{7}$ Division of General Internal Medicine, Department of Medicine, Duke University School of Medicine, Durham, NC, USA; ${ }^{8}$ Division of Geriatrics, Department of Medicine, Duke University School of Medicine, Durham, NC, USA; ${ }^{9}$ Center for the Study of Aging and Human Development, Duke University School of Medicine, Durham, NC, USA; ${ }^{10}$ Comprehensive Access and Delivery Research and Evaluation Center, lowa City Veterans Affairs Health Care System, lowa City, IA, USA; ${ }^{11}$ Iowa City Veterans Rural Health Resource Center, lowa City, IA, USA; ${ }^{12}$ Department of Psychiatry, Carver College of Medicine, University of lowa, lowa City, IA, USA; ${ }^{13}$ Department of Psychiatry and Behavioral Sciences, Duke University School of Medicine, Durham, NC, USA; ${ }^{4}$ Department of Health Policy and Management, Gillings School of Global Public Health, University of North Carolina at Chapel Hill, Chapel Hill, NC, USA.
\end{abstract}

BACKGROUND: Virtual care is critical to Veterans Health Administration (VHA) efforts to expand veterans' access to care. Health care policies such as the Veterans Access, Choice, and Accountability (CHOICE) Act and the Maintaining Internal Systems and Strengthening Integrated Outside Networks (MISSION) Act impact how the VHA provides care. Research on ways to refine virtual care delivery models to meet the needs of veterans, clinicians, and VHA stakeholders is needed.

OBJECTIVE: Given the importance of virtual approaches for increasing access to high-quality VHA care, in December 2019, we convened a Think Tank, Accelerating Implementation of Virtual Care in VHA Practice, to consider challenges to virtual care research and practice across the VHA, discuss novel approaches to using and evaluating virtual care, assess perspectives on virtual care, and develop priorities to enhance virtual care in the VHA.

METHODS: We used a participatory approach to develop potential priorities for virtual care research and activities at the VHA. We refined these priorities through forceranked prioritization and group discussion, and developed solutions for selected priorities.

RESULTS: Think Tank attendees $(n=18)$ consisted of VHA stakeholders, including operations partners (e.g., Office of Rural Health, Office of Nursing Services, Health

Prior Presentation Finding were presented at a VA Health Services Research \& Development Cyberseminar in February, 2020 (https://www. hsrd.research.va.gov/cyberseminars/catalog-archive.cfm) and at the AcademyHealth 13th Annual Conference on the Science of Dissemination and Implementation in December, 2020.

Received June 27, 2020

Accepted December 20, 2020

Published online January 26, 2021
Services Research and Development), clinicians (e.g., physicians, nurses, psychologists, physician assistants), and health services researchers. We identified an initial list of fifteen potential priorities and narrowed these down to four. The four priorities were (1) scaling evidence-based practices, (2) centralizing virtual care, (3) creating highvalue care within the VHA with virtual care, and (4) identifying appropriate patients for virtual care.

CONCLUSION: Our Think Tank took an important step in setting a partnered research agenda to optimize the use of virtual care within the VHA. We brought together research and operations stakeholders and identified possibilities, partnerships, and potential solutions for virtual care.

KEY WORDS: telemedicine; implementation science; health services research; delivery of health care.

J Gen Intern Med 36(8):2434-42

DOI: $10.1007 / \mathrm{s} 11606-020-06517-3$

(C) This is a U.S. government work and not under copyright protection in the U.S.; foreign copyright protection may apply 2021

\section{INTRODUCTION}

A critical component of a health care system such as the Veterans Health Administration (VHA) is the ability to adapt care delivery models to meet changing priorities and expectations. ${ }^{1,2}$ Policies enacted to expand veterans' access to care, such as the Veterans Access, Choice, and Accountability (CHOICE) $\mathrm{Act}^{3}$ and the Maintaining Internal Systems and Strengthening Integrated Outside Networks (MISSION) Act, ${ }^{4}$ as well as natural phenomena like pandemics, disrupt and can 
have a long-term impact on access to and delivery of health care, thus affecting how the VHA provides acute, chronic, and urgent care. Identifying how a system such as the VHA can alter its health care delivery when necessary may inform efforts by other systems to improve their access to care programs.

Compared to civilians, veterans experience a disproportionate burden of disease related to suboptimal health behaviors and social determinants of health that make access to care more challenging. ${ }^{5-8}$ Addressing the factors underlying health outcomes requires increasing the capacity for care delivered outside the traditional face-to-face encounter. ${ }^{9}{ }^{10}$ Access to care is influenced by numerous factors including patient and clinician preference and satisfaction, patient acuity and care requirements, clinical and support staffing, and available infrastructure. ${ }^{11,} 12$ Thus, new methods and models may be required to meet the VHA's ever-changing needs to ensure long-term access to care.

Although the VHA serves a unique population, it is not unique in its need to address challenges related to access to care and population health management. Like all large health systems, it must consider opportunities to enhance care, and research conducted in the VHA can significantly inform care in other health care systems in the USA and abroad. Lessons, methods, and models developed in the VHA may be especially applicable for systems with similar characteristics, such as (a) a large number of facilities requiring integration of services; (b) a focus on population health and related payment strategies; (c) the use of patient-centered medical homes; (d) a large percentage of patients with lower socioeconomic status or high mental health considerations; and (d) a need to integrate multiple clinical care, training, and research objectives.

Virtual care is a critical component of the VHA's efforts to expand veterans' access to care and to improve population health. Virtual care is defined as clinical interactions with clinicians that do not require patients and clinicians to be in the same room at the same time. ${ }^{9,13,14}$ Benefits of virtual care for clinicians include increased access for primary and specialty appointments to veterans in rural areas or with physical, psychosocial, geographical, or transportation barriers. ${ }^{15,} 16$ While the VHA relies on a developed telehealth infrastructure to facilitate patient communication with health care teams, there are disparities in technology use and access, particularly in regard to veterans who are older, living in rural areas, or of lower socioeconomic status. ${ }^{17}$ Additional research studies and improvement science projects are needed within the VHA to improve care delivery via virtual care.

We convened a 4-hour Think Tank, Accelerating Implementation of Virtual Care in VHA Practice, in December 2019 to (a) examine current virtual care research and practice, (b) consider challenges to virtual care research across the VHA, (c) discuss novel approaches to using and evaluating virtual care, and (d) assess perspectives of virtual care from a variety of stakeholders. We operationalized these aims as:

1. Identify ways to improve and advance future virtual care delivery and research in the VHA.
2. Prioritize future work and opportunities among researchoperations collaborations in the VHA.

\section{METHODS}

\section{Preparation}

In preparation for the Think Tank, we convened a planning group of seven health services researchers and two clinicians with experience in virtual care research, operations, and/or implementation science. ${ }^{18-21}$ We drafted a preliminary Think Tank agenda and identified relevant national and local VHA stakeholders to invite to the Think Tank based on our group's experience and familiarity with VHA research and operations.

Veteran Research Engagement Panel. We presented to the Veteran Research Engagement Panel (VetREP), a group of veterans and veteran caregivers who meet monthly to provide veteran-focused research input. VetREP members articulated the importance of virtual care, and that it should supplement rather than replace current VHA care. Additionally, VetREP members expressed concerns regarding insufficient veteran internet access, familiarity with virtual care tools, or ownership of newer technologies, which could exacerbate disparities in health outcomes. In response to VetREP feedback, we created three case studies demonstrating how VHA researchers and clinicians currently use their telehealth infrastructure to provide clinical care to veterans. These case studies highlighted the diversity in virtual care at the VHA and set the context for our Think Tank discussions. Overall, we relied on VetREP feedback as we framed the Think Tank questions and activities to ensure that veterans' perspectives on, comfort with, and ownership of virtual care technology were considered.

Think Tank Survey. We emailed a survey to a targeted group of local, regional, and national VHA stakeholders at 10 weeks and 7 weeks prior to the Think Tank to help us refine its agenda and discussion points. We created survey items and prepopulated responses based on opinions and insights from our group. The survey consisted of six questions and asked respondents to rank order prepopulated or write-in responses (Table 1$)$. We received a $46 \%$ response rate $(n=6 / 13)$. We used survey responses to develop overarching key questions for the Think Tank (Table 2) and to refine our format and logistics. For example, we arranged attendee seating so that both research and operations partners were included in each small group.

\section{Think Tank Activities (Fig. 1)}

Developing Potential Priorities for Virtual Care. The first activity was a 1-2-4-all liberating structures activity. ${ }^{22}$ For this 


\section{Table 1 Think Tank Survey Questions}

Please answer the following questions regarding telehealth \& virtual care. For the purposes of this survey, we define "virtual care" as any distance-based method to provide health care (e.g., telehealth, mobile health [mHealth]). Responses to these questions will help us refine the agenda and content discussed during the Think Tank.

1. Please rank the types of future research in virtual care you consider to be of greatest importance for the VHA from most important to least important.

- Staffing of virtual care

- How to sustain veteran and caregiver engagement in virtual care

- Implementation and adoption of virtual care in the VHA

- Virtual care in the context of a certain disease (please state the disease)

- Quality improvement projects

- Rapid turn-around research (e.g., study duration less than one year)

- How to identify the right population for virtual care

- Virtual care in the context of a certain population (please state the population)

- Other (please describe)

2. Please rank the types of future data in virtual care you consider to be of greatest importance to you in your role at the VHA from most important to least important.

- Data on effectiveness (please describe a specific aspect of effectiveness if pertinent)

- Qualitative \& quantitative data regarding provider and staff

perceptions/use/preferences for virtual care

- Qualitative \& quantitative data regarding veteran and caregiver perceptions/use/preferences for virtual care

- Staffing \& workforce considerations in virtual care (i.e., information on cost and number/type of staff needed in virtual care)

- Implementation/adoption/use of virtual care across facilities/integrated service networks

- Qualitative \& quantitative data regarding administrator perspectives for virtual care

- Use of virtual care in the context of a certain population (please state the population)

- Use of virtual care in the context of a certain disease (please state the disease)

- Other (please describe)

3. Please rank the needs you consider to be most important in

implementing virtual care in the VHA practice from most important to

least important.

- Greater availability of clinical staff (ie, nurses, physicians, NPs, PAs,

pharmacists)

- Guidelines/assistance in implementation

- Guidelines/assistance in research/quality improvement practices

- More research on effectiveness (please describe a specific aspect of

effectiveness if pertinent)

- More/different equipment

- Researchers who can quickly design \& complete studies and provide you with data

- More research on veteran and caregiver engagement

- Quicker timeframe between research and implementation in practice

- Greater availability of non-clinical staff (ie, schedulers, billers, coding)

- Other (please describe)

4. Please rank the barriers listed below to using virtual care within the

VHA from biggest barrier to smallest barrier.

- Not enough staffing

- Lack of evidence of clinical effectiveness

- Veterans and caregivers do not like it

- Not enough funding

- Clinical staff do not like it

- Not enough equipment

- Clinical staff do not know how to use it

- Veterans and caregivers do not know how to use it

- Lack of institutional buy-in

- Other (please describe)

5. Please rank the most important virtual care topics you want to discuss

at our Think Tank from most important to least important.

- Uptake/adoption of new virtual care programs in the VHA

- Working collaboratively across VHA offices to design/implement

virtual care programs

- Sustainability of virtual care in the VHA

- Use of virtual care in the context of a certain disease (please state the disease)

- VHA staff and administrator perceptions/use of virtual care

- Veteran and caregiver perceptions/use of virtual care

\section{Table 1. (continued)}

- Virtual care \& the MISSION Act (i.e., the changing health care payment environment)

- Use of virtual care in the context of a certain population (please state the population)

- Other (please describe)

6. If there is anything that you would like to share with us about attending our Virtual Care Think Tank, please provide that information.

VHA, Veterans Health Affairs; NP, nurse practitioner; PA, physician assistant, MISSION Act, Maintaining Internal Systems and Strengthening Integrated Outside Networks

activity, attendees were seated at tables of four people. First, each attendee had 2 minutes to reflect and brainstorm responses to two prompts: "In your role, what do you think are the top priorities for virtual care for VHA?" and "What are pressing needs for the VHA in virtual care?" Second, individuals were given 3 minutes to pair up and compare commonalities and differences in their responses. Third, two sets of pairs were allowed 4 minutes to compare responses and identify commonalities and differences. Each foursome then reported all commonalities to the larger group. While groups were reporting out, a research assistant wrote each priority on a separate large sheet of paper on the wall.

Voting on Potential Priorities. The second activity involved attendees voting on potential priorities using a modified forced-ranking prioritization method. ${ }^{23,}{ }^{24}$ Participants were given 10 votes (represented by stickers), which could be allocated to any of the 13 research priorities, with a maximum of 3 votes per item. Participants were given no specific prioritization criteria but were asked to determine, based on their perspectives, the most important unanswered priorities in

\section{Table 2 Think Tank Activities}

Key questions addressed during the Think Tank

- What are the perspectives of stakeholders including clinicians,

veterans, caregivers, non-clinical staff, and administrators regarding the use of virtual care?

- What are the characteristics of an effective virtual care program?

- What are valid clinical and non-clinical outcomes when using virtual care?

- What does an effective partnership among researchers and operations partners look like to advance virtual care use at the VHA?

- How should implementation and scalability be defined in virtual care at the VHA?

- Among the various projects, where should the VHA and related stakeholders prioritize efforts in regard to virtual care?

Developing next steps for selected priorities

Prompts

- What necessary information is needed to address this priority?

- Who are the important stakeholders to engage in addressing this

priority? (Describe global and specific stakeholders)

- What are the key clinical and organizational data needs and measurement domains that would define success?

Reporting Out

- What are the key takeaways from this priority? (Describe the importance of this priority, any patterns/conclusions that are emerging, and hypotheses that can be made)

- What are the next steps in addressing this priority? 


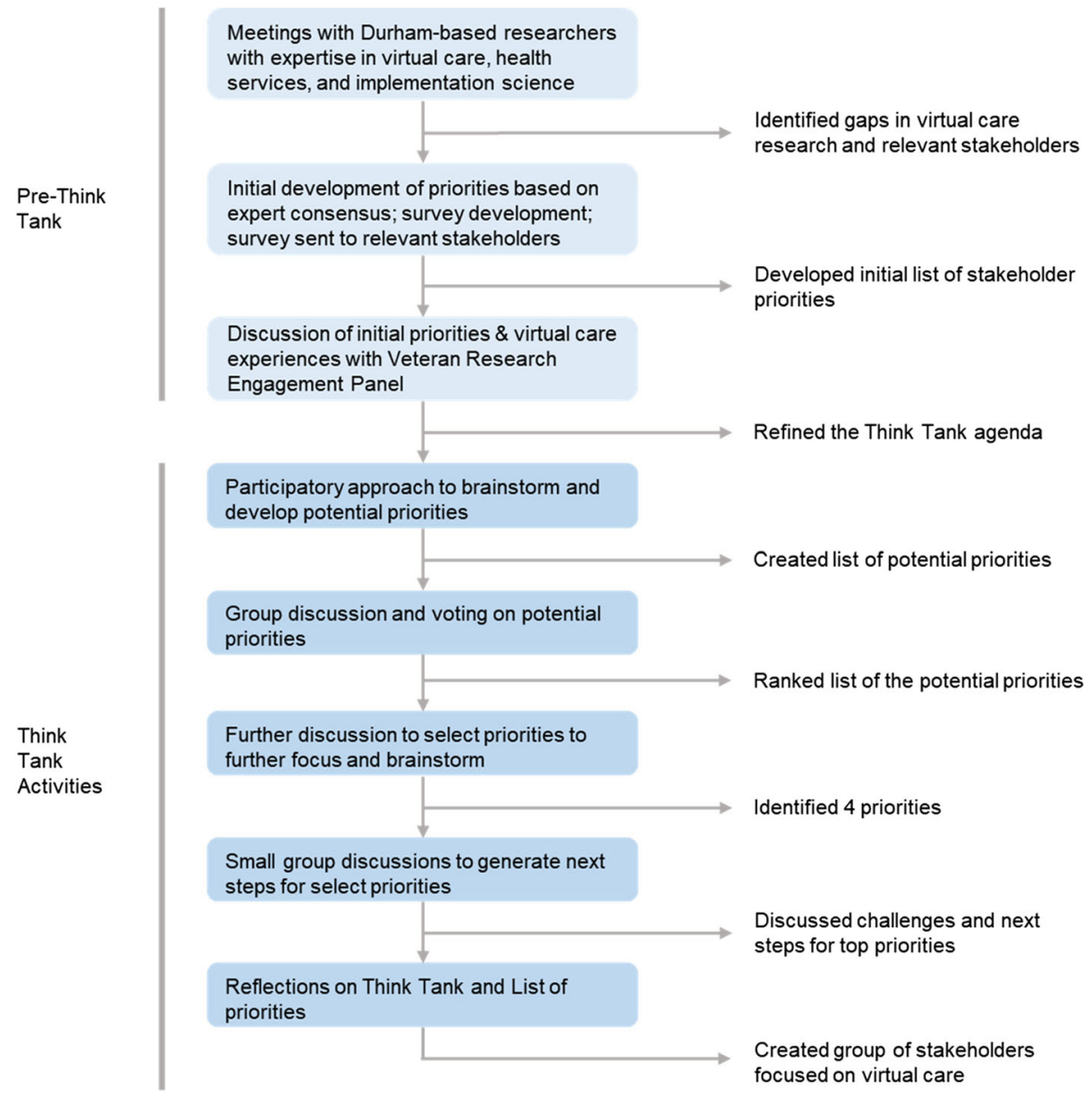

Figure 1 Order of Pre-Think Tank and Think Tank activities

virtual care for the VHA. We used color-coded stickers to indicate individuals' self-identified affiliations: primarily research, primarily operations, or both research and operations. Attendees had 20 minutes to vote for priorities by placing stickers on priority lists, after which a research assistant tallied the number of stickers on each sheet of paper. The full group discussed the rankings of priorities and selected priorities for further consideration during the remainder of the Think Tank. Although attendees could advocate for addition of a priority ranked lower than fifth or suggest removal of a priority ranked fifth or higher, we required group consensus for addition or removal of priorities.

Developing Next Steps for Selected Priorities. The final activity consisted of working in small groups to brainstorm solutions to the top four priorities. Attendees self-selected a priority for this activity. Each group was asked to articulate necessary information needed to address their specific priority in regard to stakeholders, data needs, takeaways, and next steps (Table 2). After the full group discussion, we ended the Think Tank by summarizing main takeaways and future directions for virtual care at the VHA.

\section{RESULTS}

\section{Think Tank Participants}

Think Tank participants $(n=18)$ included VHA operations partners; clinicians; researchers with expertise in health services and implementation science; and local, regional, and national administrators. Participants self-identified as primarily research $(n=10)$, primarily operations $(n=6)$, or primarily research and operations $(n=2)$.

\section{Development of Potential Priorities for Virtual Care}

We identified fifteen potential priorities (Table 3) at the completion of the first activity. During discussion, attendees posed such questions as the appropriate organizational level (i.e., system or patient) at which virtual care should exist, or how to engage vulnerable patients using virtual care modalities. Due to significant conceptual overlap, we created two categories: (1) reducing disparities and promoting equity (by combining "reducing disparities" and "promoting equity"), and (2) aligning modality and disease (by combining "aligning the virtual care modality with the clinical problem" and "patient- 
Table 3 Identified Think Tank Priorities

Priority (shortened priority)

1. How can we create valued care within the VHA with virtual care (creating high-valued care)?

2 . What virtual care services can be centralized or regional/local (deciding central, regional, or local)?

3. What are meaningful data needs and measurement domains of virtual care in the VHA (measuring virtual care)?

4. How can we take an evidence-based practice and scale it (scaling-out programs)?

5. How can we use virtual care for behavior change (changing

behaviors)?

6 . How can we educate providers to meaningfully utilize virtual care to optimize uptake (educating clinicians)?

7. *How can we use virtual care to reduce health disparities and promote equity (reducing disparities, promoting equity)?

8 . How can we share resources across the VHA to optimize care

(sharing resources)?

9. How can we triage patients for telehealth appropriateness (triaging appropriately)?

10 . **How can we align the virtual care modality with the clinical problem (aligning modality \& disease)?

11. What are the barriers to virtual care (addressing barriers)?

12. How can we integrate virtual care into the electronic health record (integrating into the EHR)?

13. How can we maximize collaboration with the Office of Connected Care and service line priorities (increasing collaboration)?

*We created this priority by combining "reducing disparities" and "promoting equity" after discussing how these two priorities shared many similarities

**We created this priority by combining "aligning the virtual care modality with the clinical problem" and "patient-level triage for telehealth appropriateness" after discussing how these two priorities shared many similarities

VHA, Veterans Health Affairs

level triage for telehealth appropriateness"). We ended the activity with thirteen priorities for virtual care.

\section{Rankings of Potential Priorities}

After voting on thirteen potential priorities, we narrowed the list to the top five priorities with the highest number of stickers (Fig. 2). Group discussion addressed (a) whether the priorities could be addressed by research, (b) the presence of available data to make operational decisions, (c) research questions and designs, (d) operational concerns, and (e) additional stakeholders who should be included in decision-making. As we determined that measuring virtual care was implicit in the other four top priorities, we removed this priority and included "data needs" as a crucial consideration in next steps. We completed this activity with four top priorities: scaling programs, centralizing virtual care, creating valued care, and aligning modality and disease.

\section{Development of Next Steps for Selected Priorities}

We identified the necessary information, key stakeholders, data needs, takeaways, and next steps (see Table 4) for four priorities.

Priority 1: How Can We Take an Evidence-Based Practice and Scale It?. The identification of core components for successful virtual care practices is essential. These components may include staffing and stakeholders, infrastructure and equipment, and data needs. For staffing, roles and responsibilities of individuals participating in the practice should be clearly articulated within the health care system (e.g., providing technical support, outlining clinician and support staff activities). Key stakeholders include other participating individuals throughout the organization (e.g., veterans, clinicians, administration) as well as process and redesign teams and local champions to promote the use of the practice. Stakeholder buyin facilitates the availability of infrastructure and equipment to promote the practice. Important data needs may include process data collected during implementation and adoption, veteran and clinician satisfaction, cost, unintended consequences, quality measures, and clinician effort and time allocation. The key takeaway is that identification of standardized virtual care processes can help decrease variability in delivery and use of virtual care.

\begin{tabular}{r|l|l|} 
& & Total \\
\hline Creating high-valued care & 26 \\
Deciding central, regional, or local & 25 \\
Measuring virtual care & 23 \\
Scaling-out programs & 20 \\
Changing behaviors & 16 \\
Educating clinicians & 14 \\
Reducing disparities, promoting equity & 11 \\
Triaging appropriately & 10 \\
Sharing resources & 10 \\
Aligning modality and disease & 9 \\
Addressing barriers & 8 \\
Integrating into the EHR & 3 \\
Increasing collaboration & 2 \\
\hline & 0
\end{tabular}
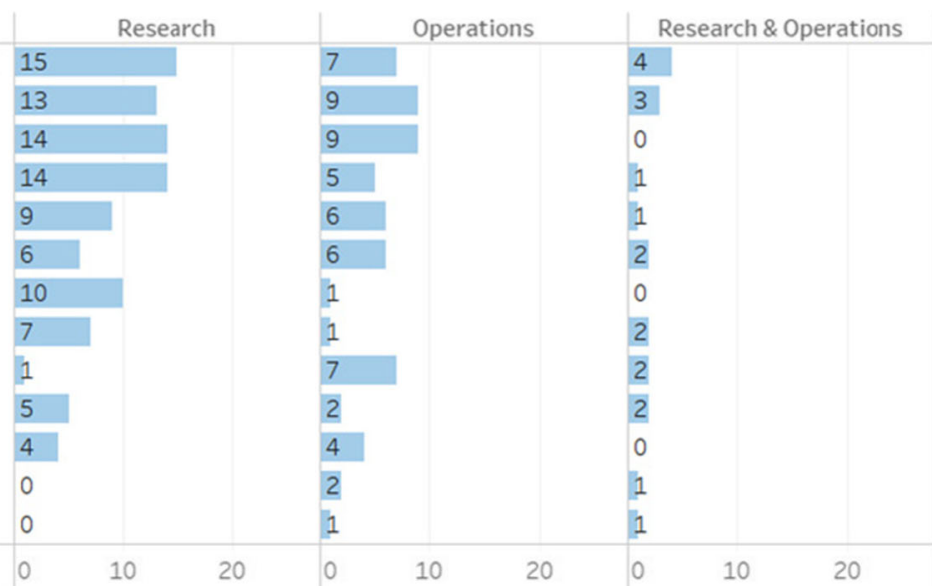

Figure 2 Rankings of potential priorities. Think Tank participants $(n=18)$ were provided with 10 stickers to vote on potential priorities. Of the 18 participants who participated in voting, 10 self-identified as primarily research, 6 self-identified as primarily operations, and 2 self-identified as primarily research and operations. EHR, electronic health record. 
Table 4 Key Areas of Information, Stakeholders, Data Needs, Takeaways, and Next Steps for 4 Priorities

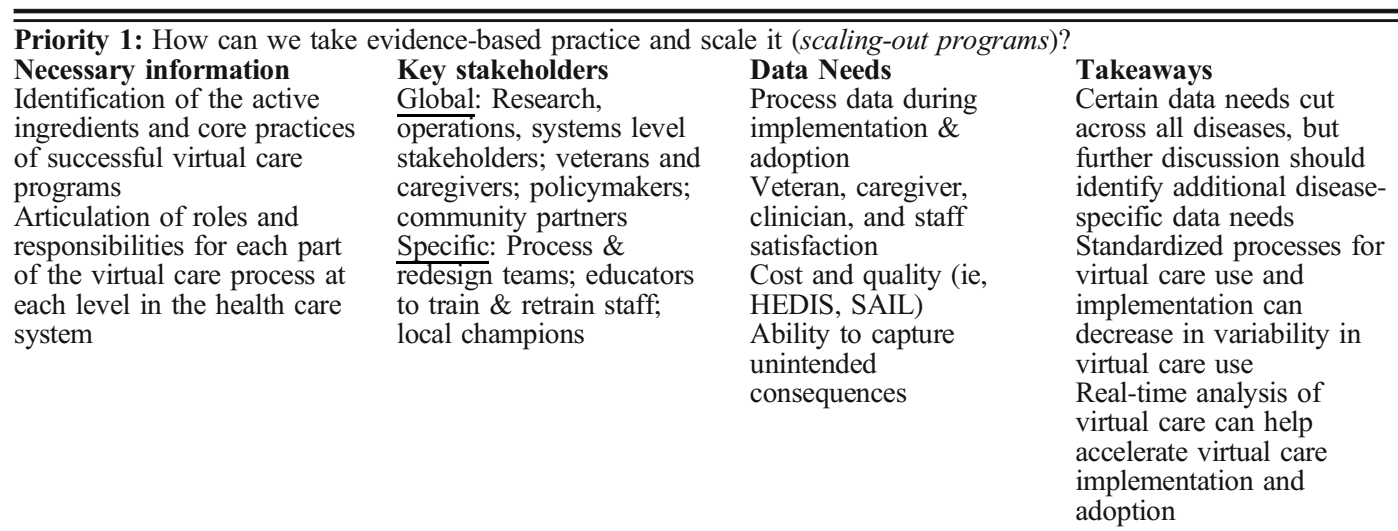

Priority 2: What virtual care services can be centralized, regional, or local (deciding central, regional, or local)? Necessary information Key stakeholders Global: Veterans and Data Needs Clarification of what services the VHA should prioritize inhouse and what services should be sent to the community

Assessment of how quality of care and veteran experience differ at each of the different directors Specific: Chief Medica Officers; VHA Central Office stakeholders

Data to address supply

Takeaways
Unclear pathways for

care

Patient reported

outcomes, quality of

care, and clinical

outcomes

Number of veterans who use VHA care for the $1^{\text {st }}$ time, and retention of veterans in VHA care decision-making make discussions about strengths and weaknesses challenging

Tailoring virtual care can occur within service areas High amount of regulation may decrease innovation and slow time to implementation

Priority 3: How can we create high-value care within the VHA with virtual care (creating high-valued care)?

\section{Necessary information}

Clarification of what parts of

virtual care are managed

locally vs. nationally

Identification of

decision-making capabilities

for initiating virtual care

Examination of effectiveness of VHA virtual care vs. community care on outcomes

\section{Key stakeholders}

Global: VHA Offices of:

Care in the Community;

Connected Care; Patient

Care services; Rural

Health; Finance; Policy and Planning

Specific: Service lines; local health system stakeholders

\section{Data Needs}

Veteran, caregiver, and clinician willingness to use, and satisfaction with, virtual care at the VHA

Veteran and caregiver satisfaction and preferences for care in/out of the VHA Impact on care, referral time, clinic volume, and documentation by specialty

\section{Takeaways}

Examination of when, how, where, for whom, and by whom virtual care is an appropriate and equitable option Identification of methods to triage appropriate veterans and service lines for virtual care

Identification of VHA's capacity and resources to meet the needs of veterans

\section{Next steps}

Identification of generalizable components of virtual care in order to develop a best practice guide to focus virtual care implementation efforts

\section{Next steps}

Discussion of how to encourage use of VHA health care services Identification of how virtual care across the VHA functions in changing funding and policy climates

\section{Next steps}

Identification of how VHA policies and procedures incentivize or disincentivize the use of virtual care Highlighting and positioning virtual care as a highly valued service at the VHA so that veterans will choose the VHA for their care

Priority 4: How can we align the virtual modality with the clinical problem (aligning modality and disease)? Necessary information Identification of resources needed (e.g., finances, clinicians, staff to assist veterans in using virtual care) to ensure appropriate use of virtual care Identification of modality of care (i.e., fixed or flexible) and end-user (i.e., specific population, individual) Defining the right patient for Key stakeholders Global: Veterans and caregivers; Service lines; VHA Central Office; and VHA Offices of: Connected Care, Rural Health, and Health Equity Specific: Local facility directors, service line leads, and information technology stakeholders

\section{Data Needs}

Continuous screening for veteran, caregiver, and clinician preferences for satisfaction with virtual care

Impact of virtual care on wait times for specialty consults and care

\section{Takeaways}

Challenges exist in aligning the patient's abilities and preferences to use virtual care, diagnosis, and the virtual care modality

Partnership between research or program offices to help inform the development, evaluation, and continued use of virtual care Developing partnerships between researchers, operations, and other stakeholders is important to use virtual care effectively

\section{Next steps}

Determination of appropriate characteristics and screening measures that can predict modality use in veterans and clinicians

Examination of how virtual care augments or replaces in-person care across disease states

HEDIS, Healthcare Effectiveness Data and Information Set; SAIL, Strategic Analytics for Improvement and Learning Value Model; VHA, Veterans Health Affairs 
Priority 2: What Virtual Care Services Can Be as Centralized or Regional/Local?. The examination of the VHA's current central, regional, and local virtual care use by service line (e.g., primary care, mental health, specialty care) is important. Data on virtual care use can inform and dictate what care is delivered, and by whom, at each level. In regard to care delivery and virtual care use, the impact of shifting health care from local patient-aligned care teams to clinicians at regional or central locations should be understood. Key stakeholders include individuals who facilitate the use of virtual care (e.g., clinicians, administrators) to provide insight into the context, available resources, and strategic directions for the VHA. Important data needs may include supply and demand for clinical care services at each level, acuity within service lines, and health and quality of care outcomes. The key takeaway is that national, regional, and local stakeholder participation, when aligned, can ensure that virtual care resources are used with equipoise to improve access to care at the VHA.

\section{Priority 3: How Can We Create High-Value Care Within the} VHA with Virtual Care?. The consideration of how to use virtual care to provide the best care to veterans, resulting in optimal outcomes, at a cost-effective and affordable price is critical. $^{25}$ Considerations may include decision-making and resource allocation. In regard to decision-making, the processes for initiating, implementing, and sustaining virtual care within the VHA at the national, regional, and local levels should be communicated clearly. Key stakeholders may include administrators throughout the organization (e.g., VHA national offices, local service line leaders and health system administrators, financial officers) as well as veteran representatives to ascertain how virtual care can be enhanced and leveraged as a service of high-value care to veterans. Involvement of key stakeholders can assist in the development of policies to triage veterans to the appropriate service lines and ensure that the VHA has the capacity and resources to meet their needs. Important data needs may include veteran satisfaction and preferences for care in/out of the VHA, clinician and veteran willingness to use virtual care, and impact of virtual care delivery on clinician staffing and resource use. The key takeaway is that clarifying which parts of virtual care are to be managed nationally, regionally, and/or locally is critical to providing high-value care in the VHA

\section{Priority 4: How Can We Align the Virtual Modality with the} Clinical Problem?. The alignment of clinical condition, appropriate virtual care modality, and veteran's abilities and preferences is significant. This alignment may include the creation of a matrix that describes the most appropriate modality (e.g., synchronous/asynchronous, telehealth, video) for various clinical conditions (e.g., diabetes, dermatologic needs, hypertension). Key stakeholders include clinicians, veterans and caregivers, and clinical service line administrators to provide insight into appropriate patient populations (e.g., clinical characteristics of veterans' situation and presentation, need to tailor to specific populations) and resources available (e.g., staff to assist veterans and clinicians in using virtual care, allocation of clinician effort for virtual care visits) to promote appropriate use of virtual care. Important data needs include veteran, caregiver, and clinician preferences for, and satisfaction with, virtual care as well as the impact of virtual care at the organizational level (e.g., referral wait times, resource allocation, staffing needs and workload). The key takeaway is that identification of the right patient, appropriate clinical need, and right virtual care modality can increase effectiveness of virtual care while ensuring optimal use of VHA virtual care resources.

\section{DISCUSSION}

The Think Tank, Accelerating Implementation of Virtual Care in VHA Practice, examined current virtual care use, discussed novel approaches to using and evaluating virtual care, and assessed perspectives of virtual care from a variety of VHA stakeholders to develop a shared understanding of VHA virtual care priorities. Leveraging the VHA's existing resources (including its integrated, nationwide telehealth infrastructure, diverse operations partners, and an embedded, robust research network) could improve health care delivery within the VHA using virtual care methods. The design and timing of projects, identification of relevant stakeholders and operations partners, and appropriate data needs were at the forefront of many Think Tank conversations. Overall, survey responses, Veteran Research Engagement Panel feedback, and Think Tank findings point to a critical need to accelerate implementation of virtual care at the VHA.

Whereas most corporations are either a health care payer or a provider of health care services, the VHA is both. A clarification of roles and duties regarding virtual care throughout the VHA would assist in developing, implementing, and adopting successful virtual care programs. Creating partnerships requires an understanding of the (a) decision-making pathway in the VHA, (b) ownership of different components of virtual care (i.e., software, hardware, staffing, data), (c) capacity of the VHA to meet supply and demand for health care, and (d) financial implications of virtual care. We began the first steps in identifying shared virtual care priorities among research and operations stakeholders by identifying and narrowing a list of potential priorities through voting, discussion, and group consensus.

Enhancing partnerships among research and operations stakeholders could address potential controversy over accepting a centralized approach or recognizing the need for adjustment to meet local fit. Research and operations partnerships can increase openness to pragmatic approaches and improve the timeliness and implementation of relevant virtual care projects. Many nationalized health care systems have 
embedded researchers responsible for examining the effectiveness and optimal use of health care programs; the VHA could glean insights from effective virtual care partnerships that have been implemented successfully in countries with nationalized health care systems. Successful partnerships can decrease the research waste of virtual care projects that do not prove successful or scalable, or which may eventually require de-implementation. As a health care system with embedded health systems researchers, the VHA is poised to excel in the development of research and operations partnerships.

There are some considerations regarding the Think Tank which should be acknowledged. Although we invited veterans and VHA stakeholders, several key individuals were unable to attend; however, our Think Tank did include national, regional, and local stakeholders with telehealth decision-making responsibilities. Virtual participation was not used, so findings should be further vetted by stakeholders, veterans, and caregivers; however, our Think Tank was expert-driven, and it benefited from bringing together national research and operations stakeholders for in-person discussions.

\section{CONCLUSION}

Virtual care is important at the VHA; however, the success and widespread use of virtual care is dependent upon implementing innovative processes, encouraging their uptake by clinicians and veterans, and developing a useful evidence base so that stakeholders can make appropriate decisions. By quickly identifying and spreading successful projects, the VHA can positively affect the care delivered to veterans. Our Think Tank took an important step in setting an agenda to optimize the use of virtual care within the VHA; in doing so, we realized that (1) clarifying who "owns" virtual care is essential to its success, and (2) creating a faster pipeline of inception to implementation can help to align aims and timelines for research and operations.

Acknowledgments The authors thank VA Mid-Atlantic Health Care Network for support of this Think Tank. The authors thank the attendees of the Think Tank: Kelli Allen, David Atkins, Hayden Bosworth, Matt Crowley, Jim Galkowski, Jennifer Gierisch, Karen Goldstein, Kaileigh Gray, Nicki Hastings, Blake Henderson, George Jackson, Jane Kim, Allison Lewinski, Michelle Lucatorto, Felicia McCant, Kathy McMullin, Leila Mureebe, Abigail Shapiro, Scott Sherman, Jennifer Strauss, Caitlin Sullivan, Matthew Tucker, Carolyn Turvey, John Whited, and Leah Zullig. The authors also thank Patrick Calhoun, Kelly Muir, Katherine Ramos, and Sarah Wilson for assistance in developing the survey and Think Tank agenda Donnalee Frega, PhD, for editorial assistance; and Eric Monson, $\mathrm{PhD}$, for data visualization assistance.

Corresponding Author: Allison A. Lewinski, PhD, MPH; HSR\&D COIN (558/152), Durham Veterans Affairs Health Care System, 508 Fulton Street, Durham, NC 27705, USA (e-mail: allison. lewinski@duke.edu).
Funding This work was supported by the Durham Center of Innovation to Accelerate Discovery and Practice Transformation grant \#CIN 13-410; Institute for Medical Research at the Durham Veterans Affairs Medical Center; the Department of Veterans Affairs Office of Academic Affiliations grant number TPH 21-OOO (to AAL); VA HSR\&D grants \#19-332 (to KDA), \#08-027 (to HBB), \#16-213 (to MJC), \#13263 (to $K M G$ ), \#18-234 (to AAL); and the Veterans Affairs Office of Rural Health (to MJC).

\section{Compliance with Ethical Standards}

Conflict of Interest Dr. Bosworth reports receiving research funds from Sanofi, Otsuka, Johnson and Johnson, Improved Patient Outcomes, Novo Nordisk, and PhRMA Foundation as well as consulting funds from Sanofi, Otsuka, Abbott, and Novartis. Dr. Zullig reports receiving funding from the PhRMA Foundation and Proteus Digital Health as well as consulting funds from Novartis. Dr. Lewinski reports receiving funding from Otsuka and the PhRMA Foundation. The remaining authors have no competing interests to declare.

Disclaimer The findings and conclusions in this document are those of the authors, who are responsible for its contents and do not represent the views of the Department of Veterans Affairs, the US Government, or Duke University; therefore, no statement in this article should be construed as an official position of the Department of Veterans Affairs or Duke University.

\section{REFERENCES}

1. Kilbourne AM, Elwy AR, Sales AE, Atkins D. Accelerating research impact in a learning health care system: VA's quality enhancement research initiative in the Choice Act era. Med Care. 2017;55 Suppl 7 Suppl 1:S4-s12.

2. Atkins D, Kilbourne AM, Shulkin D. Moving from discovery to systemwide change: the role of research in a learning health care system: experience from three decades of health systems research in the Veterans Health Administration. Annu Rev Public Health. 2017;38(1):467-487.

3. Veterans' Access to Care through Choice, Accountability, and Transparency Act of 2014. 2014. https://www.congress.gov/bill/113th-congress/ house-bill/3230. Accessed August 16th, 2020.

4. VA Maintaining Internal Systems and Strengthening Integrated Outside Networks Act of 2018. 2018. https://www.congress.gov/bill/115thcongress/senate-bill/2372/text. Accessed June 6, 2018.

5. Agha Z, Lofgren RP, VanRuiswyk JV, Layde PM. Are patients at Veterans Affairs medical centers sicker? A comparative analysis of health status and medical resource use. Arch Intern Med. 2000;160(21):32523257.

6. Selim AJ, Berlowitz DR, Fincke G, et al. The health status of elderly veteran enrollees in the Veterans Health Administration. J Am Geriatr Soc. 2004;52(8): 1271-1276.

7. Farmer CM, Hosek SD, Adamson DM. Balancing demand and supply for veterans' health care: a summary of three RAND assessments conducted under the Veterans Choice Act. Rand Health Q. 2016;6(1).

8. Sullivan JL, Davila HW, Rosen AK. The changing dynamics of providing health care to older veterans in the 21st century: how do we best serve those who have borne the battle? Public Policy Aging Rep. 2019;30(1):3-5.

9. Shulkin DJ. Beyond the VA crisis-becoming a high-performance network. N Engl J Med. 2016;374(11):1003-1005.

10. Duan-Porter W, Martinson BC, Greer N, et al. Evidence review-social determinants of health for veterans. J Gen Intern Med. 2018;33(10): 17851795.

11. Rubenstein L, Hempel S, Danz M, et al. Eight priorities for improving primary care access management in healthcare organizations: results of a modified Delphi stakeholder panel. J Gen Intern Med. 2020;35(2):523530.

12. Miake-Lye I, Mak S, Shanman R, Beroes JM, Shekelle P. Access Management Improvement: A Systematic Review. Washington (DC): Department of Veterans Affairs (US);2017. 
13. McGrail KM, Ahuja MA, Leaver CA. Virtual visits and patient-centered care: results of a patient survey and observational study. J Med Internet Res. 2017;19(5):e177.

14. Schoenfeld AJ, Davies JM, Marafino BJ, et al. Variation in quality of Urgent health care provided during commercial virtual visits. JAMA Intern Med. 2016;176(5):635-642.

15. Kehle SM, Greer N, Rutks I, Wilt T. Interventions to improve veterans access to care: a systematic review of the literature. J Gen Intern Med. $2011 ; 26(2): 689$.

16. Jacobs JC, Blonigen DM, Kimerling R, et al. Increasing mental health care access, continuity, and efficiency for veterans through telehealth with video tablets. Psychiatr Serv. 2019;70(11):976-982.

17. Duan-Porter W, Van Houtven $\mathbf{C H}$, Mahanna EP, et al. Internet use and technology-related attitudes of veterans and informal caregivers of veterans. Telemed J E Health. 2018;24(7):471-480.

18. Allen KD, Yancy WS, Jr., Bosworth HB, et al. A combined patient and provider intervention for management of osteoarthritis in veterans: a randomized clinical trial. Ann Intern Med. 2016;164(2):73-83.

19. Bosworth HB, Powers BJ, Olsen MK, et al. Home blood pressure management and improved blood pressure control: results from a randomized controlled trial. Arch Intern Med. 2011;171(13):1173-1180.
20. Crowley MJ, Edelman D, McAndrew AT, et al. Practical telemedicine for veterans with persistently poor diabetes control: a randomized pilot trial. Telemed J E Health. 2016;22(5):376-384.

21. Datta SK, Warshaw EM, Edison KE, et al. Cost and utility analysis of a store-and-forward teledermatology referral system: a randomized clinical trial. JAMA Dermatol. 2015;151(12):1323-1329.

22. Mahoney JS, Lewin L, Beeber L, Willis DG. Using liberating structures to increase engagement in identifying priorities for the APNA research council. J Am Psychiatr Nurses Assoc. 2016;22(6):504-507.

23. Gierisch JM, Myers ER, Schmit KM, et al. Prioritization of patientcentered comparative effectiveness research for osteoarthritis. Ann Intern Med. 2014;160(12):836-841.

24. Chang SM, Carey TS, Kato EU, Guise J-M, Sanders GD. Identifying research needs for improving health care. Ann Intern Med. 2012;157(6):439-445.

25. McGinnis JM, Stuckhardt L, Saunders R, Smith M. Best Care at Lower Cost: The Path to Continuously Learning Health Care in America. Washington, DC. National Academies Press; 2013.

Publisher's Note: Springer Nature remains neutral with regard to jurisdictional claims in published maps and institutional affiliations. 